\title{
THE MOTION OF A VISCOUS FLUID CONDUCTING HEAT*
}

\author{
BY \\ J. L. SYNGE \\ Dublin Institute for Advanced Studies
}

1. Introduction. The purpose of this paper is to draw attention to the comparatively simple equations governing the behaviour of a viscous compressible fluid which conducts heat. In these equations (following the suggestion of a referee who read the paper in its original and slightly different form) I use specific entropy and specific volume as basic thermodynamic variables. In this respect the argument differs from that of Eckart', who derived the equations using pressure and specific volume. I do not think that the difference is important, and I would not seek to publish my derivation of the equations were it not for the intolerable barrier which separates hydrodynamics from thermodynamics - a barrier which can be worn down only by repetition of the fact that there exist clearly formulated equations governing the behaviour of a viscous fluid conducting heat. Believing that a system of equations takes on reality only when one uses them, I have added some work on exponential solutions of the equations, linearised for the case of small disturbances from equilibrium.

Anyone who consults the compendious survey by Truesdell ${ }^{2}$ will realise that the history of the mechanics of continua is no simple thing. But it seems that $\mathrm{J}$. W. Gibbs (1839-1903) pointed the way towards the equations in question, and might have derived them eighty years ago if he had been interested in hydrodynamics. Whether the equations emerged in the interval and were lost to sight I do not know; the first derivation that I know of is that of Goldstein ${ }^{3}$, who avoided the use of entropy and thus made the work much more difficult to follow than Eckart's.

This avoidance of entropy may well be the key to the question: Why have the mechanics of continua and thermodynamics kept so far apart? For we do not find the word entropy in the indexes of the standard works of Lamb ${ }^{4}$, Loves, and Goldstein (op. cit.); nor do we find it in the index of Dryden, Murnaghan and Bateman ${ }^{6}$; MilneThomson ${ }^{7}$ introduces entropy only for a perfect gas.

There are curious time-lags in science. Maxwell's equations took some fifty years to gain full control of optics. The ideas of Gibbs have had an even greater delay in taking their due place in hydrodynamics. I believe that the cause is the same in both cases-a horror of abstraction. My guess is that the still prevalent entrophoby (to coin a word) springs from an unwritten law that mechanics should deal only with concepts directly related to ordinary experience (pressure, density, velocity, temperature, energy); in a macroscopic theory entropy must remain something rather mysterious, its physical interpretation (as a measure of disorder) coming to light only in the micro-

*Received August 13, 1954. Revised version received November 26, 1954.

${ }^{1}$ C. Eckart, Phys. Rev. (2) 58, 267-269 (1940).

${ }^{2} C^{C}$. Truesdell, J. of Rational Mechanics and Analysis 1, 125-300 (1952).

3s. Goldstein, ITodern developments in fluid dynamics, Oxford, Clarendon Press, 1938, vol. 2, pp. 601-606.

4Sir H. Lamb, Hydrod!ynamics, Cambridge I'niversity Press, 1932.

'. A. F. H. Love, The mathematical theory of elasticity, Cambridge Lniversity Press. 1934.

${ }^{6}$ Bulletin of the National Research Council, No. 84, 1932.

'L. M. Milne-Thomson, Theoretical hydrodynamirs, Macmillan, London, 1949. 
scopic view (matter resolved into molecules), a view quite out of keeping with the spirit of hydrodynamics, as ordinarily understood. However commendable this commonsense attitude may be, the price we have to pay for it in intellectual confusion is too high, and my own feeling is that thermo-hydrodynamics takes on a clear mathematical form only when entropy is given a leading role. Just what this ghostly visitor amounts to must come out $a$ posteriori (how does it behave in the mathematical theory?) and not a priori (how is it connected with our ordinary sensory perceptions?).

Entrophoby must be fought until such time as entropy takes the place it deserves in standard texts on hydrodynamics. One must keep on repeating that the equations are there and that they are understandable. Eckart's paper is only three pages long and is easily overlooked, as it apparently was by Courant and Friedrichs ${ }^{8}$, who, though otherwise commendably entrophile, refer the reader to Goldstein's derivation of the equations.

It is unfortunate that in a recent book de Groot ${ }^{9}$ has missed an opportunity to pick these equations out from surrounding thermodynamical complexities and make them readily available to mathematicians. The equations must surely be on pp. 95, 96 of his book, if we reduce the number of components to one. But I cannot find them in their entirety, since the Gibbs equation [(3.2) of the present paper] appears as an ordinary differential equation in de Groot's work, whereas to me (and surely it was so to Gibbs) it is a total differential equation in the space of the thermodynamic variables, a very different thing mathematically.

Since the purpose of this paper calls for emphasis on the simplest form of equations available, I have taken the case where there is only one coefficient of viscosity. But one can easily pass to the more general case of two coefficients, and so obtain a mathematical model closer to nature ${ }^{10}$.

2. Notation. We are concerned with Newtonian mechanics in which mass is conserved, and specific quantities will be measured per unit mass. There is something to be said for an alternative plan in which specific quantities are measured per unit standard volume (i.e. volume at standard temperature and pressure), and this is the better plan in relativity, since mass (as a form of energy) is no longer conserved. But in Newtonian mechanics the two plans are equivalent and it seems a little simpler to measure per unit mass, which is the usual way.

Latin suffixes take the values $1,2,3$, with the summation convention for repeated suffixes; $x_{i}$ are rectangular Cartesian coordinates in any Newtonian frame of reference (i.e. an unaccelerated frame), and partial derivatives with respect to the coordinates are indicated by a comma $\left(f_{, i}=\partial f / \partial x_{i}\right)$.

The following notation will be used (specific means per unit mass): $u_{i}=$ velocity, $p=$ pressure, $T=$ absolute temperature, $\rho=$ density, $\sigma=1 / \rho=$ specific rolume, $S=$ specific entropy, $U=$ specific internal energy, $\mu=$ viscosity, $\kappa=$ thermal conductivity, $E_{i j}=E_{j i}=$ stress tensor, $e_{i j}=\frac{1}{2}\left(u_{i, j}+u_{i, i}\right)=$ rate of deformation tensor, $d \tau=$ element of volume of a region of the fluid bounded by a surface $B$ always composed

${ }^{8}$ R. Courant and K. Friedrichs, Supersonic flow and shock waves, Interscience Publishers Inc., New York, 1948, p. 134. 1951.

'S. R. de Groot, Thermodynamics of irreversible processes, North-Holland Publishing Co., Amsterdam,

${ }^{10}$ See C. Truesdell, J. of Rational Mechanics and Analysis 2, 643-741 (1953); and L. Rosenhead el al., $A$ discussion on the first and second viscosities of fluids, Proc. Roy. Soc. London A226, 1-69 (1954). 
of the same particles, $d B=$ element of $B, n_{i}=$ unit outward normal to $B, X_{i}=$ specific body force, $h_{i}=$ heat-flow vector.

3. The entropy equation. The quantities $S, T, U, p$, and $\sigma$ (equivalently $\rho$ ) are thermodynamic variables. We shall select $S$ and $\sigma$ as basic thermodynamic variables; the values of $S$ and $\sigma$ at any event $(x, t)$ determine the state of the fluid at that event. We assume an equation of state

$$
U=f(S, \sigma),
$$

and further assume that the other two thermodynamic variables are also functions of $S$ and $\sigma$ consistent with the entropy equation

$$
T d S=d U+p d \sigma
$$

which is to hold for arbitrary infinitesimals $d S, d \sigma$, and thus is equivalent to

$$
T=U_{s}, \quad p=-U_{\text {o }},
$$

the subscripts denoting partial derivatives with respect to $S$ and $\sigma$.

The quantities $\mu, \kappa$ are assumed to be given positive functions of $S$ and $\sigma$.

4. The conservation of mass, the equation of momentum, and the first law of thermodynamics. The conservation of mass gives

$$
\frac{d \rho}{d t}+\rho u_{k, k}=0
$$

where for any function $F(x, t)$

$$
\frac{d F}{d t}=\frac{\partial F}{\partial t}+F_{, k} u_{k}
$$

the derivative following the fluid.

Following a portion of the fluid which consists always of the same particles, the law of momentum gives

$$
\frac{d}{d t} \int \rho u_{i} d \tau=\int \rho X_{i} d \tau+\int E_{i j} n_{i} d B
$$

and hence, since $\rho d \tau$ is conserved,

$$
\rho \frac{d u_{i}}{d t}=\rho X_{i}+E_{i i, i} .
$$

Again following the fluid, the first law of thermodynamics gives

$$
\frac{d}{d t} \int \frac{1}{2} \rho u_{i} u_{i} d \tau+\frac{d}{d t} \int \rho U d \tau=\int \rho X_{i} u_{i} d \tau+\int E_{i j} n_{j} u_{i} d B-\int h_{i} n_{i} d B,
$$

and this, when the surface integrals are changed to volume integrals and (4.4) is used, leads to

$$
\rho \frac{d U}{d t}=E_{i j} e_{i i}-h_{i, i} .
$$

5. Stress-deformation equations and thermal conduction. In listing the notation in Sec. 2 the pressure $p$ and stress $E_{i}$ were not connected. We now assume

$$
3 p=-E_{k k} \text {. }
$$


As stress-deformation equations we assume

$$
E_{i j}=-p \delta_{i j}+2 \mu\left(e_{i j}-\frac{1}{3} \delta_{i} e_{k k}\right),
$$

where $\delta_{i}$ is the Kronecker delta.

In view of (5.1) there are five equations in (5.2), not six, since the contraction $j=i$ gives an identity. On the other hand (5.2) implies (5.1).

As law of thermal conduction we assume

$$
h_{i}=-\kappa T_{. i} .
$$

6. Muster of equations. Substituting from (5.2) and (5.3) in (4.1), (4.4), and (4.6) we get the following equations:

$$
\begin{gathered}
\frac{d \rho}{d t}+\rho e_{k k}=0 \\
\rho \frac{d u_{i}}{d t}=\rho X_{i}-p_{, i}+2\left[\mu\left(e_{i j}-\frac{1}{3} \delta_{i j} e_{k k}\right)\right]_{, j}, \\
\rho \frac{d U}{d t}=-p e_{k k}+2 \mu\left(e_{i j} e_{i j}-\frac{1}{3} e_{k k}^{2}\right)+\left(\kappa T_{, i}\right)_{, i} .
\end{gathered}
$$

In these equations $e_{i j}=\frac{1}{2}\left(u_{i, j}+u_{i, i}\right)$, and we recognise here five equations for the five unknowns $u_{i}, S, \sigma$, for $\rho=1 / \sigma$ and by Sec. $3 U, T$, and $p$ are known functions of $S$ and $\sigma$. These are the desired equations governing the behaviour of a viscous fluid which conducts heat. They give a determinate problem in the sense that the number of equations equals the number of unknowns; we shall not attempt to discuss what boundary conditions are consistent with determinacy of the solution.

Equation (6.3) may be written a little differently. By (3.2), following the fluid, we have

$$
T \frac{d S}{d t}=\frac{d U}{d t}+p \frac{d \sigma}{d t}=\frac{d U}{d t}-\frac{p}{\rho} \frac{d \rho}{d t}=\frac{d U}{d t}+\frac{p}{\rho} e_{k k},
$$

and so (6.3) is equivalent to

$$
\rho T \frac{d S}{d t}=2 \mu\left(e_{i j} e_{i j}-\frac{1}{3} e_{k k}^{2}\right)+\left(\kappa T_{, i}\right)_{, i} .
$$

7. Small disturbances from equilibrium. We suppose body force absent $\left(X_{i}=0\right)$ and consider a state of equilibrium in which $u_{i}=0$ and the thermodynamic variables have constant values which we shall denote by $S^{(0)}, \sigma^{(0)}$, etc. For a small disturbance we have $u_{i}$ small and

$$
S=S^{(0)}+S^{(1)}, \quad \sigma=\sigma^{(0)}+\sigma^{(1)}, \quad \text { etc. }
$$

where the quantities marked (1) are small. We linearise the non-linear equations (6.1), (6.2), (6.5) by neglecting terms quadratic in small quantities. Thus we get

$$
\begin{gathered}
\frac{\partial \rho^{(1)}}{\partial t}+\rho^{(0)} u_{k, k}=0 \\
\rho^{(0)} \frac{\partial u_{i}}{\partial t}=-p_{, i}^{(1)}+\mu^{(0)}\left(\Delta u_{i}+\frac{1}{3} u_{k, k i}\right) \\
\rho^{(0)} T^{(0)} \frac{\partial S^{(1)}}{\partial t}=\kappa^{(0)} \Delta T^{(1)}
\end{gathered}
$$

where $\Delta$ is the Laplacian operator. 
By (3.3) we have in equilibrium

$$
T^{(0)}=U_{S}^{(0)}, \quad p^{(0)}=-U_{0}^{(0)} .
$$

For the disturbed state

$$
T^{(1)}=U_{S S}^{(0)} S^{(1)}+U_{S \sigma}^{(0)} \sigma^{(1)}, \quad p^{(1)}=-U_{\sigma S}^{(0)} S^{(1)}-U_{\sigma \sigma}^{(0)} \sigma^{(1)},
$$

where the coefficients are known constants. Since $\rho=1 / \sigma$, we have

$$
\rho^{(1)}=-\rho^{(0) 2} \sigma^{(1)} \text {. }
$$

Substitution in (7.2), (7.3), (7.4) gives the following five linear equations with constant coefficients for the determination of $u_{i}, S^{(1)}, \sigma^{(1)}$ :

$$
\left.\begin{array}{c}
\frac{\partial \sigma^{(1)}}{\partial t}-\sigma^{(0)} u_{k, k}=0 \\
\rho^{(0)} \frac{\partial u_{i}}{\partial t}-U_{\sigma S}^{(0)} S_{, i}^{(1)}-U_{\sigma \sigma}^{(0)} \sigma_{, i}^{(1)}-\mu^{(0)}\left(\Delta u_{i}+\frac{1}{3} u_{k, k i}\right)=0 \\
\rho^{(0)} T^{(0)} \frac{\partial S^{(1)}}{\partial t}-\kappa^{(0)}\left(U_{S S}^{(0)} \Delta S^{(1)}+U_{S \diamond}^{(0)} \Delta \sigma^{(1)}\right)=0 .
\end{array}\right\}
$$

8. Elementary solutions. Let us now investigate elementary solutions of (7.8) of the form

$$
\left.\begin{array}{c}
u_{i}=u_{i}^{*} \exp \left(a_{i} x_{i}+b t\right), \quad S^{(1)}=S^{*} \exp \left(a_{i} x_{i}+b t\right), \\
\sigma^{(1)}=\sigma^{*} \exp \left(a_{i} x_{i}+b t\right),
\end{array}\right\}
$$

where $u_{i}^{*}, S^{*}, \sigma^{*}, a_{i}, b$, are nine constants, in general complex. This is a general approach to the special problems considered by Lamb (op. cit., pp. 645-657).

Splitting $a_{i}$ and $b$ into real and imaginary parts,

we have

$$
a_{i}=a_{i}^{\prime}+i a_{i}^{\prime \prime}, \quad b=b^{\prime}+i b^{\prime \prime},
$$

$$
\sigma^{(1)}=\sigma^{*} \exp \left(a_{i}^{\prime} x_{i}+b^{\prime} t\right) \exp i\left(a_{i}^{\prime \prime} x_{i}+b^{\prime \prime} t\right),
$$

and similar expressions for $u_{i}$ and $S^{(1)}$; the physical disturbances are of course the real parts of these complex expressions. A realisation of the meaning of expressions like (8.3) is helped by considering the equations

$$
\begin{aligned}
a_{i}^{\prime} x_{i}+b^{\prime} t & =\text { constant }, \\
a_{i}^{\prime \prime} x_{i}+b^{\prime \prime} t & =\text { constant },
\end{aligned}
$$

each of which represents a family of moving planes (or waves), or equivalently a family of 3-flats in Newtonian space-time. It is clear that (8.4) are amplitude waves, the amplitude of the disturbance being propagated unchanged in the direction of the vector $a_{i}^{\prime}$ with velocity

$$
V_{i}^{\prime}=-a_{i}^{\prime} b^{\prime} /\left(a_{k}^{\prime} a_{k}^{\prime}\right),
$$

and (8.5) are phase waves, the phase being propagated unchanged with velocity

$$
V_{i}^{\prime \prime}=-a_{i}^{\prime \prime} b^{\prime \prime} /\left(a_{k}^{\prime \prime} a_{k}^{\prime \prime}\right) \text {. }
$$


The amplitude waves have neither wave length nor period; the wave length and period of the phase waves are

$$
\lambda=2 \pi\left(a_{i}^{\prime \prime} a_{i}^{\prime \prime}\right)^{-1 / 2}, \quad \tau=2 \pi\left|b^{\prime \prime}\right|^{-1} .
$$

The complex vector $a_{i}$ is closely related to heat flow. For the complex heat flow vector is by (7.6), to the first order,

$$
h_{i}=-\kappa^{(0)} T_{, i}^{(1)}=-\kappa^{(0)}\left[U_{S S}^{(0)} S^{*}+U_{S \sigma}^{(0)} \sigma^{*}\right] a_{i} \exp \left(a_{i} x_{i}+b t\right),
$$

and so the real heat flow vector is parallel to the planes which are parallel to the two real vectors $a_{i}^{\prime}, a_{i}^{\prime \prime}$.

We now substitute from (8.1) in (7.8), but we shall simplify the notation by dropping the label (0), so that henceforth $\rho, U_{s s}$, etc. refer to the equilibrium state. We get the following five equations:

$$
\left.\begin{array}{c}
\rho \sigma^{*} b-u_{k}^{*} a_{k}=0, \\
\rho u_{i}^{*} b-a_{i}\left(U_{\bullet s} S^{*}+U_{\bullet \cdot} \sigma^{*}\right)-\mu\left(u_{i}^{*} a_{k} a_{k}+\frac{1}{3} u_{k}^{*} a_{k} a_{i}\right)=0, \\
\rho T S^{*} b-\kappa a_{k} a_{k}\left(U_{s s} S^{*}+U_{s,} \sigma^{*}\right)=0 .
\end{array}\right\}
$$

It is convenient to subtract from the second line the first multiplied by $\frac{1}{3} \mu a_{i}$, so that our set of equations may be written

$$
\left.\begin{array}{c}
\rho b \sigma^{*}-u_{k}^{*} a_{k}=0, \\
a_{i}\left[U_{\bullet s} S^{*}+\left(U_{\bullet \bullet}+\frac{1}{3} \mu \rho b\right) \bullet^{*}\right]+u_{i}^{*}\left[\mu a_{k} a_{k}-\rho b\right]=0, \\
\rho T b S^{*}-\kappa a_{k} a_{k}\left(U_{S S} S^{*}+U_{s \sigma^{*}}\right)=0 .
\end{array}\right\}
$$

Any choice of the nine complex constants $u_{i}^{*}, S^{*}, \sigma^{*}, a_{i}, b$ satisfying these five equations gives us an elementary disturbance (8.1). To investigate the solutions of (8.11) an obvious plan is to eliminate the first five quantities, obtaining a $5 \times 5$ determinantal equation connecting $a_{i}$ and $b$, but the algebra may be reduced by using special axes.

9. Reduction of the algebraic problem. If $a_{i}$ were a real vector, we could make $a_{2}=$ $a_{3}=0$ by choosing the $x_{1}$-axis in the direction of the vector $a_{i}$. Since $a_{i}$ is in general complex, we cannot do this, but we can choose the $x_{3}$-axis perpendicular to the two real vectors $a_{i}^{\prime}, a_{i}^{\prime \prime}$ and so make

$$
a_{3}=0 \text {. }
$$

Then, with Greek suffixes for the range 1,2, our equations (8.11) read

$$
\left.\begin{array}{c}
u_{3}^{*}\left(\mu a_{\nu} a_{\nu}-\rho b\right)=0, \\
\rho b \sigma^{*}-u_{\nu}^{*} a_{\nu}=0, \\
\left.a_{\lambda}\left[C_{\sigma, \nu}\right)^{*}+\left(C_{\sigma \sigma}^{-}+\frac{1}{3} \mu \rho b\right) \sigma^{*}\right]+u_{\lambda}^{*}\left(\mu a_{\nu} a_{\nu}-\rho b\right)=0, \\
\rho T b S^{*}-\kappa a_{\nu} a_{\nu}\left(U_{s s} S^{*}+U_{S \sigma} \sigma^{*}\right)=0 .
\end{array}\right\}
$$


Elimination of $u_{\lambda}^{*}, S^{*}, \sigma^{*}$ from the last three lines gives the determinantal equation

$$
\left|\begin{array}{cccc}
a_{1} & a_{2} & 0 & \rho b \\
\rho b-\mu a_{v} a_{v} & 0 & a_{1} U_{\sigma s} & a_{1}\left(U_{\sigma \sigma}+\frac{1}{3} \mu \rho b\right) \\
0 & \rho b-\mu a_{v} a_{v} & a_{2} U_{\sigma s} & a_{2}\left(U_{\sigma \sigma}+\frac{1}{3} \mu \rho b\right) \\
0 & 0 & \rho T b-\kappa a_{v} a_{v} U_{s s} & -\kappa a_{v} a_{v} U_{s \sigma}
\end{array}\right|=0 .
$$

Introducing the notation (for any axes)

$$
A=a_{k} a_{k}, \quad C=\rho b-\mu a_{k} a_{k},
$$

so that

$$
\rho b=C+\mu A,
$$

and further writing

$$
\Pi=\kappa\left(U_{s s} U_{\theta \sigma}-U_{\sigma s}^{2}\right)-\mu T U_{\sigma \sigma}, \quad \Sigma=\mu T-{ }_{\kappa} U_{S S},
$$

the first of (9.2) and the determinantal equation (9.3) read

$$
\begin{gathered}
C u_{3}^{*}=0, \\
C\left(K_{3} C^{3}+K_{2} C^{2}+K_{1} C+K_{0}\right)=0,
\end{gathered}
$$

where

$$
\begin{aligned}
& K_{0}=A^{2}\left(\Pi-\frac{1}{3} \mu^{2} \Sigma A\right), \\
& K_{1}=-A T U_{\text {of }}+\frac{1}{3} A^{2} \mu\left(\mu T-2 \kappa U_{S S}\right), \\
& K_{2}=A\left(\frac{5}{3} \mu T-{ }_{\kappa} U_{S S}\right), \\
& K_{3}=T .
\end{aligned}
$$

We note that the complex vector $a_{i}$ appears now only in the form $A$, and we shall therefore classify all elementary solutions of the form (8.1) according to the value of $A$ as follows:

Class I: $A \neq 0, A \neq 3 \Pi /\left(\mu^{2} \Sigma\right)$,

Class II: $A=3 \Pi /\left(\mu^{2} \Sigma\right)$,

Class III: $A=0$.

Disturbances of Class I are to be regarded as the general type. By (9.7) we get four values for $C$, one of which is $C=0$; hence we get four values for $b$, one of which is

$$
b=\mu A / \rho .
$$

This means that if we choose the two real vectors $a_{i}^{\prime}, a_{i}^{\prime \prime}$ arbitrarily [except for the slight restriction (9.9)], thus fixing the planes of the amplitude waves and the phase waves, then we obtain four values of $b$, i.e. four values for the velocities of the amplitude 
waves and the phase waves. We see, from the first of (9.2), that the solution (9.12) leaves $u_{3}^{*}$ arbitrary; the other three values of $b$ make $u_{3}^{*}=0$ and hence give plane disturbances, for which also, by (8.9) and (9.1), we have $h_{3}=0$.

A disturbance of Class II makes $K_{0}=0$, and thus, of the four values of $C$, two are zero. The two non-zero values of $C$ give plane disturbances. Note that in general

$$
a_{k} a_{k}=a_{k}^{\prime} a_{k}^{\prime}-a_{k}^{\prime \prime} a_{k}^{\prime \prime}+2 i a_{k}^{\prime} a_{k}^{\prime \prime},
$$

and therefore the reality of $A$ in (9.10) implies the orthogonality of the amplitude waves and the phase waves.

For a disturbance of Class III we have $K_{0}=K_{1}=K_{2}=0$; therefore $C=0$ and hence $b=0$. This is an interesting case, since the vanishing of $b$ tells us that the disturbance is steady (independent of time). By (9.13) the vectors $a_{i}^{\prime}, a_{i}^{\prime \prime}$ are orthogonal and equal in magnitude, and if we take the axes of $x_{1}$ and $x_{2}$ along these vectors, the disturbance is of the form

$$
\sigma^{(1)}=\sigma^{*} \exp a_{1}^{\prime}\left(x_{1}+i x_{2}\right),
$$

with similar expressions for $u_{i}$ and $S^{(1)}$. Substituting these expressions in (7.8) or putting $a_{1}=1, a_{2}=i, b=0$ in (9.2), we find that $u_{i}^{*}, S^{*}, \sigma^{*}$, are to satisfy

$$
u_{1}^{*}+i u_{2}^{*}=0, \quad U_{\sigma s} S^{*}+U_{\sigma \sigma} \sigma^{*}=0 .
$$

This completes our analysis of elementary disturbances of the form (8.1), which we have here studied for their own sake without reference to boundary conditions. There is of course no implication that such solutions need hold throughout all space and for all time; we may, if we like, consider such solutions (or a superposition of them) in a limited portion of space-time, and investigate what boundary conditions are appropriate to these solutions. 